\title{
Nuclear collective tunneling between Hartree states
}

\author{
T. Kohmura \\ Department of Economics, Josai University, Sakado 350-0295, Japan \\ M. Maruyama \\ Department of Physics, Tohoku University, Sendai 980-8578, Japan \\ Y. Hashimoto \\ Institute of Physics, University of Tsukuba, Tsukuba 305-8571, Japan
}

(Received 5 December 2002; published 28 May 2003)

\begin{abstract}
We formulate the nuclear collective tunneling transition from one Hartree state to another, consistently with the Hartree states. A Hamiltonian effective for the collective tunneling as well as for Hartree states is obtained with the parameters determined by the Hartree calculations. A real-time description for the tunneling is proposed. It is shown that a nuclear system governed by the Hamiltonian symmetric between two Hartree states collectively tunnels back and forth between the two states owing to the residual interaction, so that the system makes harmonic tunneling oscillations. While a crowd of quantum fluctuations coherently shifts back and forth in phase with the tunneling oscillations of the center of mass of two wave packets, the symmetric nuclear system retains the energy for the harmonic tunneling oscillations. The collective tunneling transitions are analyzed in an adiabatic approximation.
\end{abstract}

DOI: 10.1103/PhysRevC.67.054316

PACS number(s): 21.60.-n

\section{INTRODUCTION}

A finite quantum mechanical system provides an infinite number of Hartree states with various symmetries and boundary conditions. The order formation in such a system must proceed through a series of collective transitions from one metastable state to another. Expressing metastable states in the Hartree approximation, we describe the collective tunneling transition from one Hartree state to another. The transition is induced by a residual interaction between nucleons in the present theory beyond the Hartree approximation.

In atomic and molecular physics, it has been observed that atoms coherently tunnel back and forth between two regions of classically stable motion [1]. The tunneling oscillations are stable and periodic, if the tunneling Hamiltonian is symmetric between the two regions. The mechanism of the atomic harmonic tunneling oscillations is applied to the products of atomic clocks. We will investigate the harmonic collective tunneling oscillations of nuclei.

In the previous papers, we performed the relativistic Hartree calculations [2] and the nonrelativistic meson mean-field calculations [3] to obtain the Hartree states of nuclei and developed the steering field theory for the nuclear collective tunneling transition from one Hartree state to another [4,5].

In the present paper we analyze the collective tunneling between the Hartree states, especially harmonic collective tunneling oscillations. We reform the field theoretical Hamiltonian used in the Hartree calculations into a Hamiltonian effective for the Hartree states and for the collective tunneling between the Hartree states. The Hamiltonian in the present theory beyond the Hartree approximation involves the residual interaction between nucleons. In the case of the collective tunneling from an oblate Hartree state to a prolate Hartree state, the residual interaction is a quadrupolequadrupole interaction, which is expressed in terms of the operators that constitute $\mathrm{SU}(5)$ algebra.

We formulate the collective tunneling transitions in a realtime description. The collective tunneling is expressed in terms of the motion of the center of mass of two wave packets, one on the side of the oblate Hartree state and the other on the side of the prolate Hartree state. The Hamiltonian that is symmetric in the particle-hole number space allows harmonic collective tunneling oscillations of nuclei. While a crowd of quantum fluctuations coherently shifts back and forth in phase with the tunneling oscillations of the center of mass of wave packets, the nucleus retains the energy for the harmonic tunneling oscillations. This is the mechanism for the symmetric nuclear system to maintain the harmonic tunneling oscillations.

In Sec. II, we present a Hamiltonian effective for the Hartree states and for the collective tunneling between the Hartree states. In Sec. III, the adiabatic collective tunneling approximation is developed. In Sec. IV, we analyze the harmonic collective tunneling oscillations in terms of the motion of the center of mass of two wave packets. Discussions and conclusion are given in Sec. V.

\section{HAMILTONIAN EFFECTIVE FOR HARTREE STATES AND FOR COLLECTIVE TUNNELING}

We have analyzed the nuclear Hartree states in the relativistic Hartree calculations [2] and in the nonrelativistic meson mean-field calculations [3]. In a theory beyond the Hartree approximation the Hartree states are affected by a residual interaction, which gives rise to the collective tunneling of the system from one Hartree state to another. In this paper we analyze the collective tunneling between Hartree states. It is aimed to formulate the collective tunneling transitions between the Hartree states in a real-time description and to derive a Hamiltonian effective for the collective tun- 
neling as well as for the Hartree states. The parameters in the effective Hamiltonian are determined by the Hartree calculations.

Prolate and oblate Hartree states are one of the features of the nuclei that have valence nucleons in the $s$ and $d$ major shell, $\left(s d_{2} d_{-2} d_{1} d_{-1} d_{0}\right)^{4}$, where the subscripts $\mu$ of $d_{\mu}$ stand for the $z$ component of orbital angular momentum $l$ $=2$ and the superscript 4 indicates the spin and isospin degrees of freedom for nucleons. We treat the collective tunneling transition of $s$ - and $d$-shell nuclei from the oblate Hartree state to the prolate Hartree state. A typical example of the nuclei that exhibit both an oblate and a prolate Hartree state is ${ }^{28} \mathrm{Si}$ [6]. While a configuration of nucleons in the nonspherical $d$ states deforms the Hartree potential, the nuclear residual interaction that is rotationally symmetric induces a collective tunneling transition between the two deformed Hartree states.

We formulate the collective tunneling in the description $[4,5]$ based on the nonrelativistic meson mean-field calculations for the Hartree states. The description adopts one sort of meson field in place of $\sigma$ and $\omega$ mesons and endorses the nuclear saturation by the truncation of the meson space. The nonrelativistic nuclear field Hamiltonian is written as

$$
\begin{aligned}
H_{\mathrm{nucl}}= & \int\left[\psi^{\dagger}(\vec{r})\left(-\frac{\nabla^{2}}{2 M}\right) \psi(\vec{r})+\frac{1}{2}\left\{\pi^{2}(\vec{r})\right.\right. \\
& \left.\left.+\phi(\vec{r})\left(-\nabla^{2}+m^{2}\right) \phi(\vec{r})\right\}-g \phi(\vec{r}) \psi^{\dagger}(\vec{r}) \psi(\vec{r})\right] d^{3} r,
\end{aligned}
$$

where $\psi$ is nucleon field, $\phi$ and $\pi$ are meson fields, and $M$, $m$, and $g$ are nucleon mass, meson mass, and meson-nucleon coupling constant, respectively.

Let us consider a nuclear system that has two Hartree states $\Psi_{\mathrm{i}}$ and $\Psi_{\mathrm{f}}$. The Hartree states $\Psi_{\mathrm{i}}$ and $\Psi_{\mathrm{f}}$ are specified by the meson mean fields $\varphi^{\mathrm{i}}$ and $\varphi^{\mathrm{f}}$, respectively. Using the meson mean fields $\varphi^{\mathrm{i}}$ and $\varphi^{\mathrm{f}}$ in the two Hartree states determined in the mean-field calculations, we formulate the collective tunneling between the two Hartree states. The meson mean field $\varphi$, which varies from $\varphi^{\mathrm{i}}$ to $\varphi^{\mathrm{f}}$, determines a residual interaction to steer the nuclear collective tunneling from $\Psi_{\mathrm{i}}$ to $\Psi_{\mathrm{f}}$.

Here we briefly show how to derive a Hamiltonian effective for the Hartree states and for the collective tunneling between the Hartree states $[4,5]$. In terms of the meson mean fields in the two Hartree states, we define the tunneling steering field to be

$$
\varphi_{\mathrm{s}}(\vec{r})=\frac{1}{2}\left\{\varphi^{\mathrm{f}}(\vec{r})-\varphi^{\mathrm{i}}(\vec{r})\right\},
$$

and the principal mean field to be

$$
\varphi_{\mathrm{p}}(\vec{r})=\frac{1}{2}\left\{\varphi^{\mathrm{i}}(\vec{r})+\varphi^{\mathrm{f}}(\vec{r})\right\} .
$$

Suppose that the nucleus of ${ }^{28} \mathrm{Si}$ is polarized along $z$ axis in the two deformed Hartree states. The two Hartree states, oblate and prolate, are symmetric to each other with respect to deformation parameter [3], i.e., the principal mean field is spherically symmetric,

$$
\varphi_{\mathrm{p}}(\vec{r})=\varphi_{\mathrm{p}}(r) Y_{00}(\theta, \varphi),
$$

and the tunneling steering field is of quadrupole symmetry,

$$
\varphi_{\mathrm{s}}(\vec{r})=\varphi_{\mathrm{s}}(r) Y_{20}(\theta, \varphi) \text {. }
$$

When we quantize the meson field $\phi(\vec{r})$, we truncate the meson field space by taking into account only the principal mean field $\varphi_{\mathrm{p}}(\vec{r})$, the tunneling steering field of quadrupole symmetry,

$$
\bar{\varphi}_{20}(\vec{r})=N_{2} \varphi_{\mathrm{s}}(r) Y_{20}(\theta, \varphi),
$$

and the other components of quadrupole symmetry,

$$
\bar{\varphi}_{2 m}(\vec{r})=N_{2} \varphi_{\mathrm{s}}(r) Y_{2 m}(\theta, \varphi),
$$

with the normalization factor $N_{2}$,

$$
\begin{gathered}
\phi(\vec{r})=\varphi_{\mathrm{p}}(\vec{r})+\hat{\phi}(\vec{r}), \\
\pi(\vec{r})=0,
\end{gathered}
$$

where the quantum quadrupole field is quantized as

$$
\hat{\phi}(\vec{r})=\sum_{m} \frac{1}{\sqrt{2 \omega_{2}}}\left\{a_{2 m} \bar{\varphi}_{2 m}(\vec{r})+a_{2 m}^{\dagger} \bar{\varphi}_{2 m}^{*}(\vec{r})\right\}
$$

and

$$
\omega_{2}^{2}=\left\langle\bar{\varphi}_{2 m}\left|-\nabla^{2}+m^{2}\right| \bar{\varphi}_{2 m}\right\rangle .
$$

The spherical principal mean field $\varphi_{\mathrm{p}}(\vec{r})$ is assumed to contribute only to the nuclear single-particle energies, but not to the residual interaction energies. The set of quadrupole fields $\bar{\varphi}_{2 m}$ makes the meson field rotationally invariant. The components of quadrupole with $m \neq 0$ are necessary to take into account any orientation of the deformed Hartree states in the initial and final states of the tunneling process.

The truncated meson field $\phi$ is related to the nucleon field $\psi$ by the field equation,

$$
\left(-\nabla^{2}+m^{2}\right) \phi(\vec{r})=g \psi^{\dagger}(\vec{r}) \psi(\vec{r}) .
$$

Projecting the above equation onto the quadrupole field, we express the quantum quadrupole field $\hat{\phi}$ in terms of the nucleon field:

$$
\begin{aligned}
\hat{\phi}(\vec{r}) & =\frac{1}{\omega_{2}^{2}} \sum_{m} \bar{\varphi}_{2 m}(\vec{r}) \int \bar{\varphi}_{2 m}^{*}\left(\vec{r}^{\prime}\right)\left(-\nabla^{\prime 2}+m^{2}\right) \phi\left(\vec{r}^{\prime}\right) d^{3} r^{\prime} \\
& =\frac{g}{\omega_{2}^{2}} \sum_{m} \bar{\varphi}_{2 m}(\vec{r}) \int \bar{\varphi}_{2 m}^{*}\left(\vec{r}^{\prime}\right) \psi^{\dagger}\left(\vec{r}^{\prime}\right) \psi\left(\vec{r}^{\prime}\right) d^{3} r^{\prime}
\end{aligned}
$$


Substituting these expressions for the meson field into the field Hamiltonian in Eq. (1), we obtain

$$
\begin{aligned}
H_{\mathrm{nucl}} & =\int \psi^{\dagger}(\vec{r})\left(-\frac{\nabla^{2}}{2 M}-g \varphi_{\mathrm{p}}(\vec{r})\right) \psi(\vec{r}) d^{3} r+H, \\
H= & -\frac{g^{2}}{2 \omega_{2}^{2}} \iint \sum_{m} \psi^{\dagger}(\vec{r}) \bar{\varphi}_{2 m}(\vec{r}) \psi(\vec{r}) \psi^{\dagger}\left(\vec{r}^{\prime}\right) \\
& \times \bar{\varphi}_{2 m}^{*}\left(\vec{r}^{\prime}\right) \psi\left(\vec{r}^{\prime}\right) d^{3} r d^{3} r^{\prime} .
\end{aligned}
$$

The nuclear quadrupole-quadrupole interaction $H$ is responsible for the two deformed Hartree states and for the collective tunneling transition between them.

The derived Hamiltonian is expressed in terms of the creation and annihilation operators for $s$ nucleons $\left(c_{s i}\right)$ and for $d_{\mu}$ nucleons $\left(c_{\mu i}\right)$ in the basis states determined in the single-particle potential for the spherical principal mean field:

$$
H_{\text {nucl }}=H_{\text {shell }}+H
$$

where the shell-model Hamiltonian for nucleons in the $s$ and $d$ shells is

$$
H_{\text {shell }}=\sum_{i}\left(\varepsilon_{s} c_{s i}^{\dagger} c_{s i}+\sum_{\mu} \varepsilon_{d} c_{\mu i}^{\dagger} c_{\mu i}\right) .
$$

When the valence nucleon configurations are truncated in the $s$ and $d$ shells, the quadrupole-quadrupole interaction between the $d$-shell nucleons, responsible for the deformed Hartree states and for the collective tunneling, is expressed as

$$
H=-\kappa Q^{\dagger} Q=-\kappa \sum_{m=-2}^{2} Q_{m}^{\dagger} Q_{m}=H_{0}+H_{I}
$$

with

$$
\begin{gathered}
H_{0}=-\kappa Q_{0}^{\dagger} Q_{0}, \\
H_{I}=-\kappa\left(Q_{2}^{\dagger} Q_{2}+Q_{1}^{\dagger} Q_{1}+Q_{-1}^{\dagger} Q_{-1}+Q_{-2}^{\dagger} Q_{-2}\right) .
\end{gathered}
$$

In the numerical calculations below, the single-particle energies $\varepsilon_{s}$ and $\varepsilon_{d}$ in the spherical shell-model Hamiltonian $H_{\text {shell }}$ are assumed to be degenerate $\left(\varepsilon_{s}=\varepsilon_{d}\right)$ and the spinorbit splitting for the $d$ states is ignored. The quadrupolequadrupole interaction, which is rotationally symmetric, yields the restoring force for the oblate state to tunnel to the prolate state. The quadrupole operators in the residual interaction Hamiltonian $H$ are defined to be

$$
Q_{m}=\sqrt{10} \sum_{\mu i}(2 \mu 2 m-\mu \mid 2 m)(-1)^{\mu-m} c_{\mu i}^{\dagger} c_{\mu-m i}
$$

and $i$ stands for spin and isospin of nucleons. The explicit expressions for the quadrupole operators are the following:

$$
\begin{aligned}
Q_{0}=Q_{0}^{\dagger}= & \sqrt{\frac{5}{7}} \sum_{i}\left(2 c_{2 i}^{\dagger} c_{2 i}-c_{1 i}^{\dagger} c_{1 i}-2 c_{0 i}^{\dagger} c_{0 i}-c_{-1 i}^{\dagger} c_{-1 i}\right. \\
+ & \left.2 c_{-2 i}^{\dagger} c_{-2 i}\right), \\
Q_{1}=-Q_{-1}^{\dagger}= & \sqrt{\frac{5}{7}} \sum_{i}\left(-\sqrt{6} c_{2 i}^{\dagger} c_{1 i}-c_{1 i}^{\dagger} c_{0 i}+c_{0 i}^{\dagger} c_{-1 i}\right. \\
& \left.+\sqrt{6} c_{-1 i}^{\dagger} c_{-2 i}\right), \\
Q_{2}=Q_{-2}^{\dagger}= & \sqrt{\frac{5}{7}} \sum_{i}\left(2 c_{2 i}^{\dagger} c_{0 i}+\sqrt{6} c_{1 i}^{\dagger} c_{-1 i}+2 c_{0 i}^{\dagger} c_{-2 i}\right) .
\end{aligned}
$$

The coupling constant $\kappa$ of the quadrupole-quadrupole interaction is determined by the meson mean-field overlapped with the nucleon densities:

$$
\begin{aligned}
\kappa= & \frac{g^{2}}{20 \omega_{2}^{2}} \sum_{\mu} \iint \psi_{2 \mu-m}^{*}(\vec{r}) \bar{\varphi}_{2 m}^{*}(\vec{r}) \psi_{2 \mu}(\vec{r}) \\
& \times \psi_{2-\mu+m}^{*}\left(\vec{r}^{\prime}\right) \bar{\varphi}_{2 m}\left(\vec{r}^{\prime}\right) \psi_{2-\mu}\left(\vec{r}^{\prime}\right) d^{3} r d^{3} r^{\prime} .
\end{aligned}
$$

The value of $\kappa$ that is determined in the Hartree calculations is of the order of $0.1 \mathrm{MeV}$ for the $d$-shell nucleons [3]. The $s$-shell nucleons are assumed to be free from the residual interaction $H$ in Eq. (18), but a weak residual interaction is assumed to act between $s$ and $d$ nucleons, when it is indicated.

The residual quadrupole-quadrupole interaction forms the deformed Hartree potential for the oblate Hartree state and that for the prolate Hartree state. The residual interaction concurrently induces a nuclear collective tunneling transition between the two deformed Hartree states in the present theory beyond the Hartree approximation. For simplicity, the present calculations do not include the pairing interaction, although it may play a role in the collective tunneling between the deformed Hartree states.

The orbital angular momentum operators are also expressed as

$$
L_{m}=\sqrt{10} \sum_{\mu i}(2 \mu 2 m-\mu \mid 1 m)(-1)^{\mu-m} c_{\mu i}^{\dagger} c_{\mu-m i}
$$

and their explicit expressions are

$$
\begin{gathered}
L_{0}=\sum_{i}\left(2 c_{2 i}^{\dagger} c_{2 i}+c_{1 i}^{\dagger} c_{1 i}-c_{-1 i}^{\dagger} c_{-1 i}-2 c_{-2 i}^{\dagger} c_{-2 i}\right), \\
L_{1}=-\sum_{i}\left(\sqrt{2} c_{2 i}^{\dagger} c_{1 i}+\sqrt{3} c_{1 i}^{\dagger} c_{0 i}+\sqrt{3} c_{0 i}^{\dagger} c_{-1 i}\right. \\
\left.+\sqrt{2} c_{-1 i}^{\dagger} c_{-2 i}\right),
\end{gathered}
$$




$$
L_{-1}=\sum_{i}\left(\sqrt{2} c_{1 i}^{\dagger} c_{2 i}+\sqrt{3} c_{0 i}^{\dagger} c_{1 i}+\sqrt{3} c_{-1 i}^{\dagger} c_{0 i}+\sqrt{2} c_{-2 i}^{\dagger} c_{-1 i}\right) .
$$

The operators $Q_{m}$ and $L_{m^{\prime}}$ satisfy the commutation relation,

$$
\left[Q_{m}, L_{m^{\prime}}\right]=-\sqrt{6}\left(2 m 1 m^{\prime} \mid 2 m+m^{\prime}\right) Q_{m+m^{\prime}},
$$

which is identical to that for $\mathrm{SU}(3)$ by Elliott [7]. The commutation relation between $Q_{m}$ and $Q_{m^{\prime}}$ involves not only $L_{m+m^{\prime}}$ as in the case of SU(3) but also octupole operators $S_{m+m^{\prime}}$. Thus, the algebra of the 24 operators $c_{\mu i}^{\dagger} c_{\mu^{\prime} i}$ that appear in the present Hamiltonian $H$ is extended to $\mathrm{SU}(5)$ [8], which employs, in addition to $L_{m}$ and $Q_{m}$, the octupole and hexadecapole operators,

$$
\begin{aligned}
& S_{m}=\sqrt{10} \sum_{\mu i}(2 \mu 2 m-\mu \mid 3 m)(-1)^{\mu-m} c_{\mu i}^{\dagger} c_{\mu-m i}, \\
& R_{m}=\sqrt{10} \sum_{\mu i}(2 \mu 2 m-\mu \mid 4 m)(-1)^{\mu-m} c_{\mu i}^{\dagger} c_{\mu-m i} .
\end{aligned}
$$

The commutation relations of these operators for $\mathrm{SU}(5)$ algebra are expressed in terms of $6 j$ symbols. If we express these operators as

$$
O_{l m}= \begin{cases}L_{m}, & l=1 \\ Q_{m}, & l=2 \\ S_{m}, & l=3 \\ R_{m}, & l=4,\end{cases}
$$

the commutation relations are

$$
\begin{aligned}
{\left[O_{l_{1} m_{1}}, O_{l_{2} m_{2}}\right]=} & \sum_{l\left(=l_{1}+l_{2}+\text { odd }\right)}(-1)^{l} 2 \sqrt{10\left(2 l_{1}+1\right)\left(2 l_{2}+1\right)} \\
& \times\left\{\begin{array}{ccc}
2 & 2 & l \\
l_{1} & l_{2} & 2
\end{array}\right\}\left(l_{1} m_{1} l_{2} m_{2} \mid l m_{1}+m_{2}\right) \\
& \times O_{l m_{1}+m_{2}} .
\end{aligned}
$$

The Casimir operator for SU(5) is defined as

$$
C=L^{\dagger} L+Q^{\dagger} Q+S^{\dagger} S+R^{\dagger} R .
$$

The present interaction Hamiltonian $H=-\kappa Q^{\dagger} Q$ in Eq. (18) commutes with the Casimir operator $C$,

$$
[H, C]=0 .
$$

Therefore, eigenstates of the Hamiltonian $H$ are simultaneously eigenstates of the Casimir operator $C$. Eigenstates with the same eigenvalue as the Casimir operator $C$ constitute a representation of SU(5). Any states of a nuclear system of nucleons in the $d$ states can be classified by the representations of the Casimir operator $C$. Since the interaction Hamiltonian $H=-\kappa Q^{\dagger} Q$ is rotationally symmetric, we obtain
TABLE I. The eigenvalues $E_{k}$ of the Hamiltonian $H=-\kappa Q^{\dagger} Q$ for a two-nucleon system are listed in units of the coupling constant $\kappa$. Nuclear states with angular momentum $L$ are classified by the dimensions of $\mathrm{SU}(5)$ representations and by the eigenvalues $\lambda_{k}^{2}$ of the Casimir operator $C$.

\begin{tabular}{cccc}
\hline \hline$L$ & Dimension & $\lambda_{k}^{2}$ & $E_{k}$ \\
\hline 0 & 15 & 112.0 & -40.0 \\
1 & 10 & 72.0 & -30.0 \\
2 & 15 & 112.0 & -15.7 \\
3 & 10 & 72.0 & -8.6 \\
4 & 15 & 112.0 & -25.7 \\
\hline \hline
\end{tabular}

$$
\left[H, L_{0}\right]=0, \quad\left[H, L_{ \pm 1}\right]=0, \quad\left[H, \vec{L}^{2}\right]=0 .
$$

The eigenstates of the Hamiltonian $H$ are also simultaneously eigenstates $|L, M\rangle$ of the orbital angular momentum operators $\vec{L}^{2}$ and $L_{0}$. The eigenvalues of the Hamiltonian $H$ depend on the representation of $\mathrm{SU}(5)$ and on the angular momentum $L$, but not on $M$.

Now we demonstrate that the present SU(5) Hamiltonian $H=-\kappa Q^{\dagger} Q$ in Eq. (18) has many appropriate aspects for realistic systems. To this end, we apply the Hamiltonian to two- and four-nucleon systems for the moment. Although the Hamiltonian does not include the pairing interaction for simplicity, the qualitative features of the present discussion may be persistent even when the pairing interaction is taken into account.

First we consider a system of two nucleons in the $d$ states. We solve the eigenequations for $H$ and $C$,

$$
\begin{aligned}
& H \Psi_{k}=E_{k} \Psi_{k}, \\
& C \Psi_{k}=\lambda_{k}^{2} \Psi_{k} .
\end{aligned}
$$

The eigenvalues $E_{k}$ of the Hamiltonian $H=-\kappa Q^{\dagger} Q$ for a two-nucleon system are listed in Table I. We see in the table that the eigenvalue $E_{k}$ of the Hamiltonian increases with the compound angular momentum $L$ of the two nucleons, consistently with the nuclear spectroscopy due to short-ranged nucleon-nucleon interactions, except for the energy $E_{k}$ for $L=4$ state that is lower than the energy for $L=2$. The

TABLE II. The nuclear states of four nucleons in the $d$ states are classified by the dimensions of SU(5) representations. The column headed as "Multiplicity" shows the multiplicity of the representations with a same dimension for four-nucleon systems. Each representation contains the nuclear states specified by their angular momenta $L$.

\begin{tabular}{lcc}
\hline \hline Dimension & Multiplicity & $L$ \\
\hline 105 & 3 & $7,6,5,5,4,4,3,3,3,2,2,1,1$ \\
70 & 1 & $8,6,5,4,4,2,2,0$ \\
50 & 2 & $6,4,4,3,2,2,0,0$ \\
45 & 3 & $5,4,3,3,2,1,1$ \\
5 & 1 & 2 \\
\hline \hline
\end{tabular}


TABLE III. The eigenvalues $E_{k}$ of the Hamiltonian $H=-\kappa Q^{\dagger} Q$ in units of the coupling constant $\kappa$ and the expansion coefficients $c_{l_{12} l_{34} 0}$ of the eigenstates $\Psi_{k}=\sum c_{l_{12} l_{34} 0}\left|l_{12} l_{34} 0\right\rangle$ are listed for the four-nucleon states with $L=0$ that are classified by the dimensions of SU(5) representations and by the eigenvalues $\lambda_{k}^{2}$ of the Casimir operator $C$.

\begin{tabular}{ccccccccc}
\hline \hline$L$ & Dimension & $\lambda_{k}^{2}$ & $E_{k}$ & $c_{110}$ & $c_{330}$ & $c_{000}$ & $c_{220}$ & $c_{440}$ \\
\hline 0 & 50 & 168.0 & -93.978 & 0.962 & 0.274 & 0.000 & 0.000 & 0.000 \\
0 & 50 & 168.0 & -14.593 & -0.274 & 0.962 & 0.000 & 0.000 & 0.000 \\
0 & 50 & 168.0 & -93.978 & 0.000 & 0.000 & -0.552 & -0.216 & 0.805 \\
0 & 50 & 168.0 & -14.593 & 0.000 & 0.000 & -0.478 & 0.873 & -0.094 \\
0 & 70 & 288.0 & -102.857 & 0.000 & 0.000 & 0.683 & 0.436 & 0.586 \\
\hline \hline
\end{tabular}

nuclear states of the two-nucleon system are classified by the 15 and 10 dimensional representations of SU(5).

Second, we apply the present Hamiltonian $H=-\kappa Q^{\dagger} Q$ in Eq. (18) to a system of four nucleons in the $d$ states. Table II shows that 625 nuclear states of four nucleons in the $d$ states are classified by the $105,70,50,45$, and 5 dimensional representations of $\mathrm{SU}(5)$ as $5 \times 5 \times 5 \times 5=105 \times 3+70+50$ $\times 2+45 \times 3+5$. Taking into account the spin and isospin of nucleons, we consider below the case of a four-nucleon system in which nucleons 1 and 2 among the four nucleons have a unique combination of spin and isospin and nucleons 3 and 4 also have another unique combination of spin and isospin. Therefore, the orbital wave functions for the nucleons 1 and 2 are antisymmetrized and those for the nucleons 3 and 4 are also antisymmetrized. While the orbital wave functions of each pair of nucleons are antisymmetrized with the compound angular momentum $l=1$ or 3 , the orbital wave func- tion for the whole four-nucleon system is the antisymmetrized wave function for the nucleons 1 and 2 multiplied by that for the nucleons 3 and 4 . We see below that the nuclear states in the present system comprise one 50 dimensional, one 45 dimensional, and the singlet 5 dimensional representation of SU(5).

Expressing the compound orbital angular momentum of the nucleons 1 and 2 as $l_{12}$ and that of the nucleons 3 and 4 as $l_{34}$, we denote the orbital state of the whole four-nucleon system with angular momentum $L$ as $\left|l_{12} l_{34}, L\right\rangle$. To start the demonstration of four-nucleon states, we first consider all the nuclear states with $L=0$, including the states with even quantum numbers for $l_{12}$ and $l_{34}$. The Hamiltonian $H=-\kappa Q^{\dagger} Q$ and the Casimir operator $C$ are expressed by the matrices with the numerically calculated matrix elements on the basis of the five $L=0$ nuclear states, i.e., $|11,0\rangle$, $|33,0\rangle,|00,0\rangle,|22,0\rangle$, and $|44,0\rangle$ :

$$
H=\kappa\left(\begin{array}{lllll}
-88.000 & -20.949 & 0.000 & 0.000 & 0.000 \\
-20.949 & -20.571 & 0.000 & 0.000 & 0.000 \\
0.000 & 0.000 & -80.000 & -35.777 & 0.000 \\
0.000 & 0.000 & -35.777 & -35.102 & -8.762 \\
0.000 & 0.000 & 0.000 & -8.762 & -96.327
\end{array}\right)
$$

and

$$
C=\left(\begin{array}{rrrrr}
168.000 & 0.000 & 0.000 & 0.000 & 0.000 \\
0.000 & 168.000 & 0.000 & 0.000 & 0.000 \\
0.000 & 0.000 & 224.000 & 35.777 & 48.000 \\
0.000 & 0.000 & 35.777 & 190.857 & 30.666 \\
0.000 & 0.000 & 48.000 & 30.666 & 209.143
\end{array}\right)
$$

The simultaneous eigenstates of the Casimir operator $C$ and of the Hamiltonian $H=-\kappa Q^{\dagger} Q$ for $L=0$ are listed in Table III. Four states among five $L=0$ states belong to the two-fold 50 dimensional representations, while one state belongs to the 70 dimensional representation. The eigenvalue $\lambda_{k}^{2}$ of the Casimir operator $C$ for the two 50 dimensional representations is uniquely 168.0. Each of the twofold 50 dimensional representations contains two $L=0$ states. Note that the two $L=0$ states in one 50 dimensional SU(5) representation are split in their energies as $E_{k}=-93.978 \kappa$ and $E_{k}=-14.593 \kappa$. The eigenstates of the Hamiltonian $H$ that have the same eigenvalue $E_{k}$, but which belong to different 
TABLE IV. The eigenvalues $E_{k}$ of the Hamiltonian $H$ $=-\kappa Q^{\dagger} Q$ in units of the coupling constant $\kappa$ and the expansion coefficients $c_{l_{12} l_{34} L}$ of the eigenstates $\Psi_{k}=\sum c_{l_{12} l_{34} L}\left|l_{12} l_{34} L\right\rangle$ are listed for the $L \neq 0$ nuclear states of the present four-nucleon system. The nuclear states are classified by the dimensions of SU(5) representations and by the eigenvalues $\lambda_{k}^{2}$ of the Casimir operator $C$. It is assumed that in the present four-nucleon system the nucleons 1 and 2 have a unique combination of spin and isospin and the nucleons 3 and 4 have another unique combination of spin and isospin.

\begin{tabular}{cccccccc}
\hline \hline$L$ & Dimension & $\lambda_{k}^{2}$ & $E_{k}$ & $c_{11 L}$ & $c_{13 L}$ & $c_{31 L}$ & $c_{33 L}$ \\
\hline 1 & 45 & 128.0 & -54.428 & 0.897 & 0.000 & 0.000 & 0.442 \\
1 & 45 & 128.0 & -11.286 & -0.442 & 0.000 & 0.000 & 0.897 \\
2 & 50 & 168.0 & -78.571 & 0.864 & -0.346 & -0.346 & 0.115 \\
2 & 50 & 168.0 & -27.143 & 0.306 & 0.490 & 0.490 & 0.653 \\
2 & 45 & 128.0 & -32.857 & 0.000 & 0.707 & -0.707 & 0.000 \\
2 & 5 & 48.0 & -10.000 & -0.400 & -0.374 & -0.374 & 0.748 \\
3 & 50 & 168.0 & -40.000 & 0.000 & 0.707 & -0.707 & 0.000 \\
3 & 45 & 128.0 & -50.528 & 0.000 & 0.693 & 0.693 & 0.198 \\
3 & 45 & 128.0 & -15.187 & 0.000 & -0.140 & -0.140 & 0.980 \\
4 & 50 & 168.0 & -51.863 & 0.000 & 0.692 & 0.692 & 0.204 \\
4 & 50 & 168.0 & -13.851 & 0.000 & -0.144 & -0.144 & 0.979 \\
4 & 45 & 128.0 & -22.857 & 0.000 & 0.707 & -0.707 & 0.000 \\
5 & 45 & 128.0 & -15.714 & 0.000 & 0.000 & 0.000 & 1.000 \\
6 & 50 & 168.0 & -18.571 & 0.000 & 0.000 & 0.000 & 1.000 \\
\hline \hline
\end{tabular}

50 dimensional representations, have a same structure of the wave functions, while the wave functions are composed by different orders of combinations of the angular momenta $l$ $=2$ of the four nucleons. In the present case that the nucleons 1 and 2 have a unique combination of spin and isospin and the nucleons 3 and 4 have also another unique combination of spin and isospin, only the two basis states $|11,0\rangle$ and $|33,0\rangle$ are allowed among the five $L=0$ basis states, i.e., $|11,0\rangle,|33,0\rangle,|00,0\rangle,|22,0\rangle$, and $|44,0\rangle$ : Only one of the twofold 50 dimensional representations is allowed for the present nuclear orbital states. The $L=0$ eigenstate of the Hamiltonian with eigenvalue $E_{k}=-93.978 \kappa$ belonging to the allowed 50 dimensional representation is the ground state of the present nuclear system.

Next we consider nuclear states with $L \neq 0$ in the case that the nucleons 1 and 2 among the four nucleons have a unique combination of spin and isospin and the nucleons 3 and 4 have another unique combination of spin and isospin. The eigenvalues of the Casimir $C$ and of the Hamiltonian $H=-\kappa Q^{\dagger} Q$ are listed in Table IV. Their simultaneous eigenstates in the present case are classified into the three representations, i.e., the 50, 45, and 5 dimensional representations of $\mathrm{SU}(5)$.

We show in Fig. 1 the energy levels of the present fournucleon system. The energy levels belonging to the $50 \mathrm{di}$ mensional representation form a ground band, while those belonging to the 45 and 5 dimensional representations form sidebands. The set of lowest-lying energy levels with $L$ $=0,2,4$, and 6 in the ground band features a rotational band structure. Note that this structure of energy levels appropriate for realistic systems is obtained from the Hamil-

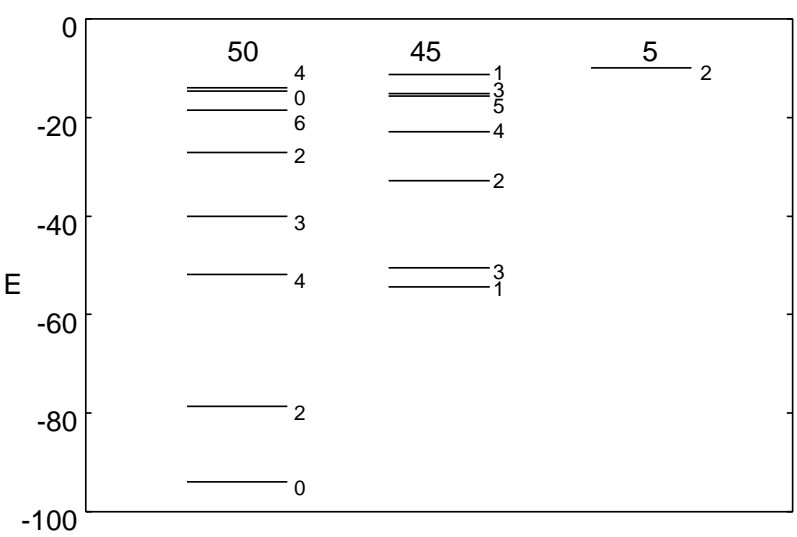

FIG. 1. The energy levels for a four-nucleon system are shown. In the present system, it is assumed that one pair of nucleons have a unique combination of spin and isospin and the other pair of nucleons also have another unique combination of spin and isospin. The nuclear states of the present four-nucleon system are classified by the 50,45 , and 5 dimensional representations of SU(5). The integral number shown aside each energy level represents the angular momentum $L$ for the nuclear state. The nuclear energies $E$ are shown in units of $\frac{5}{7} \kappa$.

tonian in Eq. (18) that takes into account the quadrupolequadrupole interaction, although the present Hamiltonian does not include the pairing interaction.

\section{ADIABATIC COLLECTIVE TUNNELING APPROXIMATION}

Now we analyze the nuclear collective tunneling transition from one Hartree minimum state to another. A typical example of the collective tunneling transitions between two Hartree states is of ${ }^{28} \mathrm{Si}$ nucleus [6], which has 12 valence nucleons in the $s$ and $d$ major shells. A nuclear system with 12 valence nucleons in the $s$ and $d$ major shells has two Hartree minimum states, i.e., an oblate and a prolate deformed state.

We analyze a collective tunneling transition from the oblate state to the prolate state of a 12-nucleon system in an adiabatic approximation to the effective Hamiltonian in Eq. (16). The Hamiltonian takes into account the quadrupolequadrupole residual interaction. The residual interaction that is rotationally symmetric gives rise to the restoring force for the oblate state to tunnel to the prolate state. It is assumed that the nucleus is polarized along the $z$ axis in the two deformed Hartree states.

The Hartree states are eigenstates of the Hartree Hamiltonian for $H_{0}$ in Eq. (19),

$$
H_{\text {Hartree }}=-\kappa\left\langle Q_{0}\right\rangle Q_{0} .
$$

According to the variation of nuclear quadrupole moment $\left\langle-Q_{0}\right\rangle$ during the tunneling transition from the oblate state to the prolate state, the single-particle energies $\varepsilon_{\mu}$ for the $d_{\mu}$ states vary, while the single-particle energy levels cross each other. Therefore, in the oblate Hartree state with a negative nuclear quadrupole moment, $\left\langle-Q_{0}\right\rangle<0$, eight nucleons among the 12 valence nucleons in the $s$ and $d$ major shells 
TABLE V. The nuclear quadrupole moments $\left\langle-Q_{0}\right\rangle$, the adiabatic nuclear energies $\epsilon_{2 n}$, and the single-particle energies $\varepsilon_{\mu}$ in the adiabatic $2 n$-particle- $2 n$-hole states $|2 n\rangle,\left|4, \theta_{4}=0\right\rangle$, and $\mid 4, \theta_{4}$ $=\pi / 2\rangle$ are listed. The values of the energies are shown in units of $\frac{5}{7} \kappa$ and the values of the nuclear quadrupole moments are in units of $\sqrt{\frac{5}{7}}$.

\begin{tabular}{lcccccc}
\hline \hline & & \multicolumn{5}{c}{$\varepsilon_{\mu}$} \\
\cline { 3 - 7 }$|2 n\rangle$ & $\left\langle-Q_{0}\right\rangle$ & $\epsilon_{2 n}$ & $d_{ \pm 2}$ & $s$ & $d_{ \pm 1}$ & $d_{0}$ \\
\hline$|0\rangle$ & -16.0 & -256.0 & -32.0 & 0.0 & 16.0 & 32.0 \\
$|2\rangle$ & -10.0 & -100.0 & -20.0 & 0.0 & 10.0 & 20.0 \\
$\left|4, \theta_{4}=0\right\rangle$ & -4.0 & -16.0 & -8.0 & 0.0 & 4.0 & 8.0 \\
$\left|4, \theta_{4}=\pi / 2\right\rangle$ & 4.0 & -16.0 & 8.0 & 0.0 & -4.0 & -8.0 \\
$|6\rangle$ & 10.0 & -100.0 & 20.0 & 0.0 & -10.0 & -20.0 \\
$|8\rangle$ & 16.0 & -256.0 & 32.0 & 0.0 & -16.0 & -32.0 \\
\hline \hline
\end{tabular}

fill the single-particle states $d_{2}$ and $d_{-2}$, while in the prolate state with $\left\langle-Q_{0}\right\rangle>0$, eight nucleons fill the states $d_{1}$ and $d_{-1}$ on the other hand. The other four nucleons vary the occupying states in the space of the $s$ and $d_{0}$ states during the tunneling transition.

We define "particles" for the $d_{1}$ and $d_{-1}$ single-particle states and "holes" for the $d_{2}$ and $d_{-2}$ states. In the present adiabatic approximation, we assume that the nucleus makes a transition from the oblate zero-particle-zero-hole Hartree state $|0\rangle$ to the prolate eight-particle-eight-hole Hartree state $|8\rangle$, taking the transitional states of nucleon configurations $\left(d_{2} d_{-2}\right)^{4-n}\left(d_{1} d_{-1}\right)^{n}$ with $n=1,2$, and 3 in four steps during the tunneling transition. The transitional nuclear states in the nucleon configurations $\left(d_{2} d_{-2}\right)^{4-n}\left(d_{1} d_{-1}\right)^{n}$ in intermediate states during the transition are denoted as $2 n$-particle$2 n$-hole states $|2 n\rangle$. This model is called the adiabatic collective tunneling model.

The configurations of nucleons in the $s$ and $d_{0}$ states in the adiabatic $2 n$-particle- $2 n$-hole states $|2 n\rangle$ are determined by minimizing the adiabatic nuclear energies for the Hartree Hamiltonian in Eq. (42). Expressing the $2 n$-particle- $2 n$-hole states as

$$
\begin{aligned}
\left|2 n, \theta_{2 n}\right\rangle= & \prod_{i}(-1)^{n}\left(\cos \theta_{2 n} c_{s i}^{\dagger}+\sin \theta_{2 n} c_{0 i}^{\dagger}\right) \\
& \times\left(c_{2}^{\dagger} c_{-2}^{\dagger}\right)^{4-n}\left(c_{1}^{\dagger} c_{-1}^{\dagger}\right)^{n}|0\rangle
\end{aligned}
$$

and minimizing the energies

$$
\begin{aligned}
\epsilon_{2 n}\left(\theta_{2 n}\right)= & \left\langle 2 n, \theta_{2 n}\left|H_{\text {Hartree }}\right| 2 n, \theta_{2 n}\right\rangle \\
= & -\frac{5 \kappa}{7}\left\{(16-6 n)^{2}\right. \\
& \left.-16(15-6 n) \sin ^{2} \theta_{2 n}+48 \sin ^{4} \theta_{2 n}\right\},
\end{aligned}
$$

we obtain the parameters $\theta_{2 n}$ for single-particle state mixing of $s$ and $d_{0}$ nucleons and the adiabatic nuclear energies $\epsilon_{2 n}$ for the $2 n$-particle- $2 n$-hole states $|2 n\rangle$.

The above variation for the adiabatic nuclear energies $\epsilon_{2 n}$ determines the single-particle states of the nucleons in the $s$ and $d_{0}$ states to be the $s$ state for $\theta_{2 n}=0$ when the nucleus is at the phase of the zero-particle-zero-hole and two-particletwo-hole configurations with the nuclear quadrupole moment $\left\langle-Q_{0}\right\rangle<0$ and the single-particle states to be the $d_{0}$ state for $\theta_{2 n}=\pi / 2$ when the nucleus is at the phase of the sixparticle-six-hole and eight-particle-eight-hole configurations with $\left\langle-Q_{0}\right\rangle>0$. When the nucleus is at the phase of the four-particle-four-hole configuration, the two adiabatic states, $\left|4, \theta_{4}=0\right\rangle$ with $\left\langle-Q_{0}\right\rangle<0$ and $\left|4, \theta_{4}=\pi / 2\right\rangle$ with $\left\langle-Q_{0}\right\rangle>0$, are degenerate in their energies. The adiabatic nuclear energies $\epsilon_{2 n}$ for $n=1,2$, and 3 provide a potential energy barrier between the two Hartree states, i.e., the zeroparticle-zero-hole state $|0\rangle$ and the eight-particle-eight-hole state $|8\rangle$, that have the minimum adiabatic energies $\epsilon_{0}$ $=\epsilon_{8}$. There also exists a potential energy barrier between the two states, $\left|4, \theta_{4}=0\right\rangle$ and $\left|4, \theta_{4}=\pi / 2\right\rangle$. These two degenerate states are coupled to each other by a coupling between the nucleons in the $s$ and $d_{0}$ states. The coupling gives rise to a minor tunneling transition of the nuclear system from $\mid 4, \theta_{4}$ $=0\rangle$ to $\left|4, \theta_{4}=\pi / 2\right\rangle$, even when the coupling is weak. Using the configurations of the $s$ and $d_{0}$ nucleons determined by the adiabatic energy variation for Eq. (44), we define the adiabatic $2 n$-particle- $2 n$-hole states $|2 n\rangle$. The four-particlefour-hole state is adiabatically determined to be

$$
|4\rangle=\frac{1}{\sqrt{2}}\left(\left|4, \theta_{4}=0\right\rangle+\left|4, \theta_{4}=\pi / 2\right\rangle\right)
$$

by taking into account a weak coupling between the $s$ and $d_{0}$ nucleons.

The calculated adiabatic energies $\epsilon_{2 n}$ of the $2 n$-particle$2 n$-hole states $|2 n\rangle,\left|4, \theta_{4}=0\right\rangle$, and $\left|4, \theta_{4}=\pi / 2\right\rangle$, are listed in Table V. The energies of the two Hartree states $|0\rangle$ and $|8\rangle$ are degenerate with the minimum adiabatic energies $\epsilon_{0}=\epsilon_{8}$ $=-\frac{5}{7} 256 \kappa$. While the adiabatic energies $\epsilon_{2 n}$ are symmetric with respect to $2 n<4$ and $2 n>4$, the energy $\epsilon_{4}$ of the fourparticle-four-hole state is at the top of the potential energy barrier between the two Hartree states $|0\rangle$ and $|8\rangle$. The nucleus in either one of the two adiabatic four-particle-fourhole states $\left|4, \theta_{4}=0\right\rangle$ and $\left|4, \theta_{4}=\pi / 2\right\rangle$ with the degenerate adiabatic energies $\epsilon_{4}=-\frac{5}{7} 16 \kappa$ is not spherical, but is deformed with the nuclear quadrupole moment indicated in Table V, respectively. Note that the nucleus does not proceed through a spherical state during the collective tunneling transition from the oblate state to the prolate state. Thus, we see that the quantum mechanical view of collective tunneling transition is different from the classical view. The latter assumes that the nuclear system makes a transition from the oblate state to the prolate state through a spherical state at the top of the potential energy barrier, if it proceeds on a shortest way. The energy at the top of the potential energy barrier in the quantum mechanical view is lower than the energy $\epsilon$ $=0$ in the spherical nuclear state at the top of the potential energy barrier in the classical view.

Now we analyze the collective tunneling transition of the present nuclear system from the oblate zero-particle-zero- 
TABLE VI. The eigenvalues $E_{k}$ of $H_{\text {adi }}$ in unit of $\frac{5}{7} \kappa$ and the expansion coefficients $c_{2 n}^{k}$ of the eigenstates $\left|\Psi_{k}\right\rangle=\Sigma_{n} c_{2 n}^{k}|2 n\rangle$ in terms of the adiabatic $2 n$-particle- $2 n$-hole states $|2 n\rangle$ are listed. The upper and lower signs in the double signs of the coefficients $c_{2 n}^{k}$ stand for the smaller and larger of the two values of $2 n$ in the coefficients $c_{2 n}^{k}$, respectively.

\begin{tabular}{ccccc}
\hline \hline$k$ & $E_{k}$ & $c_{0,8}^{k}$ & $c_{2,6}^{k}$ & $c_{4}^{k}$ \\
\hline 0 & -271.3 & 0.671 & 0.213 & 0.085 \\
1 & -269.6 & $\mp 0.680$ & $\mp 0.193$ & 0.000 \\
2 & -128.2 & -0.213 & 0.567 & 0.515 \\
3 & -86.4 & \pm 0.193 & $\mp 0.680$ & 0.000 \\
4 & 27.4 & 0.062 & -0.364 & 0.853 \\
\hline \hline
\end{tabular}
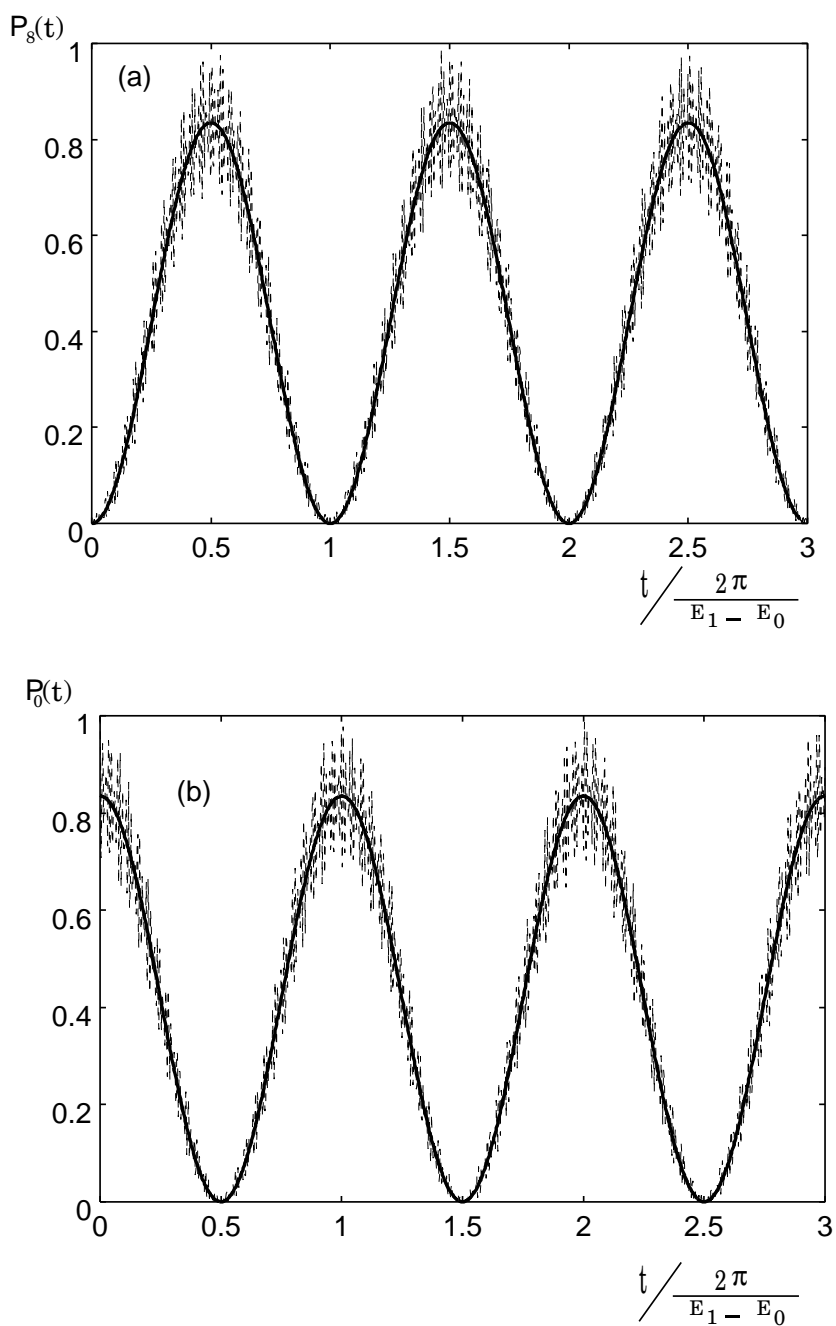

FIG. 2. The probabilities (a) $P_{8}(t)$ of the nuclear collective tunneling transition starting with the Hartree state $|0\rangle$ to the counterpart Hartree state $|8\rangle$ and (b) $P_{0}(t)$ of the return tunneling transition to the initial Hartree state $|0\rangle$ calculated in the whole space (dotted line) and in the two-state approximation (solid line) are shown as a function of time $t$ in units of the period $\tau=2 \pi /\left(E_{1}\right.$ $\left.-E_{0}\right)$ of the harmonic tunneling oscillations. The parts cover three cycles of the collective tunneling oscillations. The initial value of $P_{0}(t)$ in the two-state approximation is $P_{0}(0)=\mid \Sigma_{k=0}^{1}\left\langle 0 \mid \Psi_{k}\right\rangle$ $\times\left.\left\langle\Psi_{k} \mid 0\right\rangle\right|^{2}=0.834$. hole Hartree state $|0\rangle$ to the prolate eight-particle-eight-hole Hartree state $|8\rangle$ through the adiabatic $2 n$-particle- $2 n$-hole states $|2 n\rangle$ with $n=1,2$, and 3 in four steps. The Hamiltonian $H=-\kappa Q^{\dagger} Q$ in Eq. (18) is adiabatically expressed by the $5 \times 5$ tridiagonal matrix on the basis of the $2 n$-particle$2 n$-hole states $|2 n\rangle$,

$$
H_{\text {adi }}=-\frac{5 \kappa}{7}\left(\begin{array}{ccccc}
256 & 48 & & & 0 \\
48 & 100 & 36 \sqrt{2} & & \\
& 36 \sqrt{2} & 16 & 36 \sqrt{2} & \\
& & 36 \sqrt{2} & 100 & 48 \\
0 & & & 48 & 256
\end{array}\right) \text {, }
$$

with the matrix elements

$$
H_{\text {adi } n n^{\prime}}=\left\langle 2 n|H| 2 n^{\prime}\right\rangle .
$$

The calculated eigenvalues $E_{k}$ and eigenstates $\Psi_{k}$ of the Hamiltonian $H_{\text {adi }}$ are listed in Table VI. The energies of two ground states are nearly degenerate with $E_{1}=-\frac{5}{7} 269.6 \kappa$ and $E_{0}=-\frac{5}{7} 271.3 \kappa$. These two nearly degenerate eigenstates are mainly composed of an odd and an even linear combination, respectively, of the zero-particle-zero-hole state $|0\rangle$ and the eight-particle-eight-hole state $|8\rangle$.

The time evolution of the nuclear state during a tunneling transition starting with the oblate zero-particle-zero-hole Hartree state $|0\rangle$ is expressed in a real-time description as

$$
\Psi(t)=e^{-i H_{\mathrm{adi}} t}|0\rangle=\sum_{k} e^{-i E_{k} t}\left|\Psi_{k}\right\rangle\left\langle\Psi_{k} \mid 0\right\rangle .
$$

The probability of the nuclear transition to $2 n$-particle$2 n$-hole state $|2 n\rangle$ is

$$
\begin{aligned}
P_{2 n}(t)= & \mid\langle 2 n| e^{-\left.i H_{\mathrm{adi}^{t}}|0\rangle\right|^{2}} \\
= & \mid \sum_{k}\left\langle 2 n \mid \Psi_{k}\right\rangle e^{-\left.i E_{k^{t}}\left\langle\Psi_{k} \mid 0\right\rangle\right|^{2}} \\
= & \sum_{k k^{\prime}}\left\langle 0 \mid \Psi_{k}\right\rangle\left\langle\Psi_{k} \mid 2 n\right\rangle\left\langle 2 n \mid \Psi_{k^{\prime}}\right\rangle\left\langle\Psi_{k^{\prime}} \mid 0\right\rangle \\
& \times \cos \left\{\left(E_{k}-E_{k^{\prime}}\right) t\right\} \\
= & \delta_{n 0}-2 \sum_{k k^{\prime}}\left\langle 0 \mid \Psi_{k}\right\rangle\left\langle\Psi_{k} \mid 2 n\right\rangle\left\langle 2 n \mid \Psi_{k^{\prime}}\right\rangle \\
& \times\left\langle\Psi_{k^{\prime}} \mid 0\right\rangle \sin ^{2}\left(\frac{E_{k}-E_{k^{\prime}}}{2} t\right) .
\end{aligned}
$$

We demonstrate the calculated probability $P_{8}(t)$ of the transition to the prolate eight-particle-eight-hole Hartree state $|8\rangle$ and the probability $P_{0}(t)$ of the return transition to the initial zero-particle-zero-hole Hartree state $|0\rangle$ in Fig. 2, which shows that the transition probabilities $P_{0}(t)$ and $P_{8}(t)$ grossly oscillate with a long period of $\tau=2 \pi /\left(E_{1}-E_{0}\right)$, fluctuating with short periods of quantum fluctuations. Note that the frequency of the tunneling oscillations is propor- 
tional to the energy splitting of the two nearly degenerate ground states. Since the energy splitting is an exponentially small quantity, it takes a long time for the oblate state to tunnel to the prolate state.

If we take only the contribution of the two nearly degenerate ground states $\Psi_{k}$ with $k=0$ and 1 in the above expression for $P_{2 n}(t)$ (two-state approximation), the transition probabilities $P_{0}(t)$ and $P_{8}(t)$ feature only one mode of oscillations (ground state oscillations) with the unique period $\tau=2 \pi /\left(E_{1}-E_{0}\right)$, removing the quantum fluctuations. We show in Fig. 2 the transition probabilities $P_{0}(t)$ and $P_{8}(t)$ smoothed by the two-state approximation.
In the case that the Hamiltonian $H$ is symmetric with respect to the particle-hole number $2 n$, the even and odd overlapping of the Hartree states with the eigenstates $\Psi_{k}$,

$$
\begin{aligned}
& \left\langle\Psi_{0} \mid 0\right\rangle=\left\langle\Psi_{0} \mid 8\right\rangle=-\left\langle\Psi_{1} \mid 0\right\rangle=\left\langle\Psi_{1} \mid 8\right\rangle, \\
& \left\langle\Psi_{2} \mid 0\right\rangle=\left\langle\Psi_{2} \mid 8\right\rangle=-\left\langle\Psi_{3} \mid 0\right\rangle=\left\langle\Psi_{3} \mid 8\right\rangle,
\end{aligned}
$$

leads to matching and mismatching relations of the matrix elements in a good approximation. The overlapping relations simplify the probability of the collective tunneling from the Hartree state $|0\rangle$ to the counterpart Hartree state $|8\rangle$ as

$$
\begin{aligned}
P_{8}(t)= & 4\left|\left\langle 0 \mid \Psi_{1}\right\rangle\left\langle\Psi_{0} \mid 0\right\rangle\right|^{2} \sin ^{2}\left(\frac{E_{1}-E_{0}}{2} t\right) \\
& +\left.8\left\langle 0 \mid \Psi_{2}\right\rangle\left\langle\Psi_{0} \mid 0\right\rangle\right|^{2} \sin \left(\frac{E_{1}-E_{0}}{2} t\right) \sin \left(\frac{E_{3}-E_{2}}{2} t\right) \cos \left\{\frac{\left(E_{3}+E_{2}\right)-\left(E_{1}+E_{0}\right.}{2} t\right\}+\cdots
\end{aligned}
$$

On the right hand side of the above equation the first line represents the tunneling oscillations $\sin ^{2}\left[\left(E_{1}-E_{0}\right) / 2 t\right]$ between the two ground states $\Psi_{k}$ with $k=0$ and 1 and the second line represents the quantum fluctuations $\cos \left\{\left[\left(E_{3}\right.\right.\right.$ $\left.\left.\left.-E_{2}\right)-\left(E_{1}-E_{0}\right)\right] / 2 t\right\}$. Thus, the transition probabilities $P_{8}(t)$ and $P_{0}(t)$ are composed of collective tunneling oscillations between the two ground states with the long period $\tau$ $=2 \pi /\left(E_{1}-E_{0}\right)$ and of quantum fluctuations with a relatively short period. The tunneling probabilities feature ground state oscillations plus quantum fluctuations.

The quantum fluctuations are dominated by two modes of beat with frequencies $\left(E_{1}-E_{0}\right) / 2 \pi$ and $\left(E_{3}-E_{2}\right) / 2 \pi$. Owing to the beat with the frequency $\left(E_{1}-E_{0}\right) / 2 \pi$, we in fact see in Fig. 2 that the quantum fluctuations in $P_{8}(t)$ are most evident at a time $t=\left(n+\frac{1}{2}\right) \tau$, i.e.,

$$
\left(E_{1}-E_{0}\right) t=(2 n+1) \pi,
$$

when the transition probability $P_{8}(t)$ is the maximum, while the quantum fluctuations almost completely vanish at a time $t=n \tau$, i.e.,

$$
\left(E_{1}-E_{0}\right) t=2 n \pi,
$$

when the transition probability $P_{8}(t)$ is the minimum. The probability $P_{0}(t)$ has a similar feature that the quantum fluctuations almost completely vanish at a time $t=\left(n+\frac{1}{2}\right) \tau$ when the transition probability $P_{0}(t)$ is the minimum. Thus, a crowd of quantum fluctuations coherently shifts back and forth in phase with the collective tunneling oscillations.

The period of the collective tunneling oscillations is exponentially long, while the periods of other nuclear oscillations are relatively short: Nonadiabatic transitions may take place a short while after the system starts tunneling. Thus the system retains the energy for collective tunneling oscillations. This is the mechanism for the symmetric nuclear sys- tem to maintain the collective tunneling oscillations. This fact must be persistent even when we take into account a larger space of adiabatic particle-hole states or the effects of nonadiabatic transition.

\section{HARMONIC COLLECTIVE TUNNELING OSCILLATIONS}

In the preceding section we analyzed the nuclear collective tunneling transition from the oblate Hartree state to the prolate Hartree state, using the adiabatic collective tunneling approximation. The adiabatic approximation provides a tridiagonal expression for the Hamiltonian for collective tunneling. When the nuclear system completes one tunneling transition from one Hartree state to another, it starts to make a return tunneling transition to the initial Hartree state. In fact, the calculated result for the transition probabilities in the preceding section suggests that the nuclear system governed by the Hamiltonian $H_{\text {adi }}$ in Eq. (46) makes harmonic collective tunneling oscillations with the period $\tau=2 \pi /\left(E_{1}\right.$ $-E_{0}$ ). In this section we argue that the tridiagonal Hamiltonian symmetric with respect to the particle-hole number $2 n$ allows harmonic collective tunneling oscillations of the system. We express the harmonic collective tunneling in terms of the motion of the center of mass of wave packets.

The tridiagonal Hamiltonian is, in general, expressed on the basis of adiabatic $2 n$-particle- $2 n$-hole states $|2 n\rangle$ :

$$
\begin{gathered}
H=H_{0}+H_{I}, \\
H_{0}=\sum_{n=0}^{N} \epsilon_{n}|2 n\rangle\langle 2 n|,
\end{gathered}
$$




$$
H_{I}=\sum_{n=0}^{N-1}\left\{h_{n n+1}|2 n\rangle\left\langle 2(n+1)\left|+h_{n+1 n}\right| 2(n+1)\right\rangle\langle 2 n|\right\},
$$

where the maximum number of particles and holes is denoted as $2 N$. We assume the symmetry with respect to $2 n$ $<N$ and $2 n>N$, i.e., $\epsilon_{n}=\epsilon_{N-n}$ and $h_{n n \pm 1}=h_{N-n N-n \mp 1}$. The adiabatic $2 n$-particle- $2 n$-hole energies $\epsilon_{n}$ provide a potential energy barrier between the zero-particle-zero-hole Hartree state $|0\rangle$ and the $2 N$-particle- $2 N$-hole Hartree state $|2 N\rangle$.

In order to analyze the harmonic oscillations of the collective tunneling transition starting with the Hartree state $|0\rangle$, we formulate the time evolution of the number of particles and holes in the nuclear system. The nuclear wave function expressed in terms of the adiabatic $2 n$-particle$2 n$-hole states $|2 n\rangle$,

$$
\Psi(t)=e^{-i H t}|0\rangle=\sum_{n}|2 n\rangle\left\langle 2 n\left|e^{-i H t}\right| 0\right\rangle,
$$

is split into two wave packets by the potential energy barrier, one on the side of the zero-particle-zero-hole state $|0\rangle$ and the other on the side of the $2 N$-particle- $2 N$-hole state $|2 N\rangle$. Although no wave packets can traverse the potential energy barrier, the current flows from one wave packet to another through the barrier, varying the balance of the two wave packets. Defining the density matrix

$$
P_{n}=|2 n\rangle\langle 2 n|
$$

and the current for the nuclear system to vary the state from an adiabatic state $|2 n\rangle$ to $|2(n+1)\rangle$ [4],

$$
j_{n+1 n}=-i\left\{h_{n+1 n}|2(n+1)\rangle\left\langle 2 n\left|-h_{n n+1}\right| 2 n\right\rangle\langle 2(n+1)|\right\},
$$

in fact, we can prove the continuity relation,

$$
-\frac{\partial}{\partial t} P_{n}=-i\left[H, P_{n}\right]=j_{n+1 n}-j_{n n-1} .
$$

The time evolution of the number of particles and holes is formulated as the motion of the center of mass of the two wave packets in the particle-hole number space. The coordinate of the center of mass of the two wave packets at time $t$ is expressed in terms of the eigenstates $\Psi_{k}$ and the eigenenergies $E_{k}$ of the Hamiltonian $H$ as

$$
\begin{aligned}
\nu(t) & =\langle\Psi(t)|\hat{\nu}| \Psi(t)\rangle \\
& =\sum_{n}\left\langle 0\left|e^{i H t}\right| 2 n\right\rangle 2 n\left\langle 2 n\left|e^{-i H t}\right| 0\right\rangle \\
& =\sum_{k k^{\prime} n}\left\langle 0 \mid \Psi_{k}\right\rangle\left\langle\Psi_{k} \mid 2 n\right\rangle 2 n\left\langle 2 n \mid \Psi_{k^{\prime}}\right\rangle\left\langle\Psi_{k^{\prime}} \mid 0\right\rangle e^{i\left(E_{k}-E_{k^{\prime}}\right) t},
\end{aligned}
$$

where the operator for the coordinate of the center of mass of wave packets is defined as

$$
\hat{\nu}=\sum_{n=0}^{N} 2 n|2 n\rangle\langle 2 n|
$$

The velocity operator for the center of mass of wave packets is defined by the Heisenberg equation of motion as

$$
\begin{aligned}
\hat{\nu} & =i[H, \hat{\nu}] \\
& =2 i \sum_{n=0}^{N}\left\{h_{n n+1}|2 n\rangle\left\langle 2(n+1)\left|-h_{n+1 n}\right| 2(n+1)\right\rangle\langle 2 n|\right\} .
\end{aligned}
$$

The acceleration operator for the center of mass is also defined by the equation of motion,

$$
\begin{aligned}
\ddot{\nu}= & i[H, \hat{\nu}] \\
= & 2 \sum_{n=0}^{N}\left[( \epsilon _ { n + 1 } - \epsilon _ { n } ) \left\{h_{n n+1}|2 n\rangle\langle 2(n+1)|\right.\right. \\
& \left.+h_{n+1 n}|2(n+1)\rangle\langle 2 n|\right\} \\
& \left.+2\left(h_{n n+1} h_{n+1 n}-h_{n n-1} h_{n-1 n}\right)|2 n\rangle\langle 2 n|\right] .
\end{aligned}
$$

We now analyze the harmonic tunneling oscillations, expressing the Hamiltonian $H$ in Eq. (55) in terms of the eigenstates $\Psi_{k}$ of $H$ as

$$
H=\sum_{k} E_{k}\left|\Psi_{k}\right\rangle\left\langle\Psi_{k}\right|
$$

and using the operator for the coordinate of the center of mass of wave packets relative to the center $|2 n\rangle=|N\rangle$ of the oscillation region,

$$
\hat{\nu}_{r}=\sum_{n=0}^{N}(2 n-N)|2 n\rangle\langle 2 n| .
$$

The equation of motion of the center of mass is written as

$$
\begin{aligned}
\ddot{\nu}_{r}= & -\left[H,\left[H, \hat{\nu}_{r}\right]\right] \\
= & -\left\{\sum_{k k^{\prime}} E_{k}^{2}\left(\left|\Psi_{k}\right\rangle\left\langle\Psi_{k}\left|\hat{\nu}_{r}\right| \Psi_{k^{\prime}}\right\rangle\left\langle\Psi_{k^{\prime}}|+| \Psi_{k^{\prime}}\right\rangle\left\langle\Psi_{k^{\prime}}\left|\hat{\nu}_{r}\right| \Psi_{k}\right\rangle\left\langle\Psi_{k}\right|\right)\right. \\
& \left.-2 \sum_{k k^{\prime}} E_{k} E_{k^{\prime}}\left|\Psi_{k}\right\rangle\left\langle\Psi_{k}\left|\hat{\nu}_{r}\right| \Psi_{k^{\prime}}\right\rangle\left\langle\Psi_{k^{\prime}}\right|\right\} \\
= & -\sum_{k<k^{\prime}}\left(E_{k}-E_{k^{\prime}}\right)^{2}\left(\left|\Psi_{k}\right\rangle\left\langle\Psi_{k}\left|\hat{\nu}_{r}\right| \Psi_{k^{\prime}}\right\rangle\right. \\
& \left.\times\left\langle\Psi_{k^{\prime}}|+| \Psi_{k^{\prime}}\right\rangle\left\langle\Psi_{k^{\prime}}\left|\hat{\nu}_{r}\right| \Psi_{k}\right\rangle\left\langle\Psi_{k}\right|\right) .
\end{aligned}
$$

The two-state approximation, which takes into account only the two nearly degenerate ground states $\Psi_{k}$ with $k=0$ and 1 , leads to 


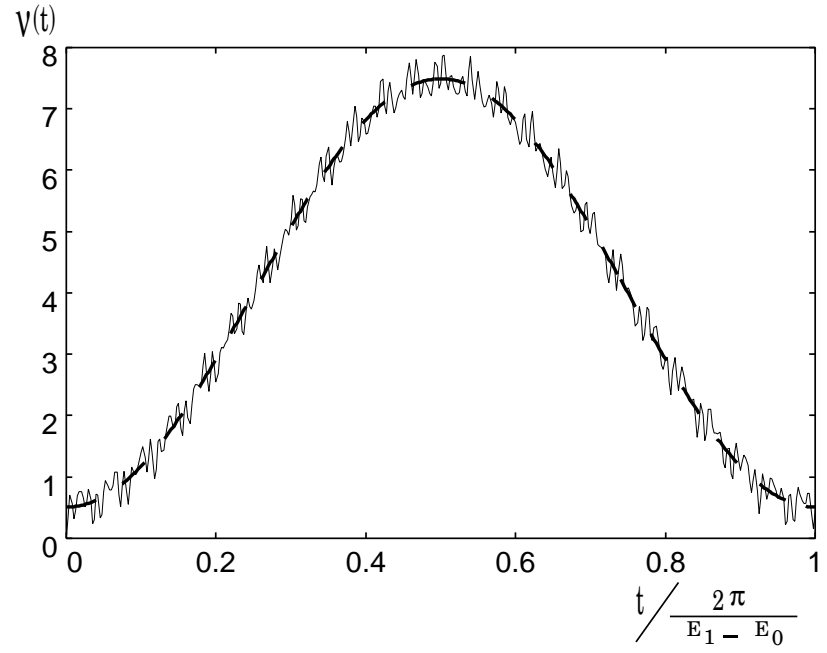

FIG. 3. The values of the coordinate $\nu(t)=\nu_{r}(t)+4$ of the center of mass of the two wave packets in the 12-nucleon system governed by the adiabatic Hamiltonian $H_{\text {adi }}$ in Eq. (46), calculated in the whole space (solid line) and in the two-state approximation (dashed line), are shown as a function of time $t$ in units of the period $\tau=2 \pi /\left(E_{1}-E_{0}\right)$ of the harmonic tunneling oscillations. The motion of the center of mass features harmonic oscillations in the two-state approximation calculation.

$$
\begin{aligned}
\ddot{\hat{\nu}}_{r}= & -\left(E_{1}-E_{0}\right)^{2}\left(\left|\Psi_{1}\right\rangle\left\langle\Psi_{1}\left|\hat{\nu}_{r}\right| \Psi_{0}\right\rangle\left\langle\Psi_{0}|+| \Psi_{0}\right\rangle\right. \\
& \left.\times\left\langle\Psi_{0}\left|\hat{\nu}_{r}\right| \Psi_{1}\right\rangle\left\langle\Psi_{1}\right|\right) \\
= & -\left(E_{1}-E_{0}\right)^{2} \sum_{k k^{\prime}=0}^{1}\left|\Psi_{k}\right\rangle\left\langle\Psi_{k}\left|\hat{\nu}_{r}\right| \Psi_{k^{\prime}}\right\rangle\left\langle\Psi_{k^{\prime}}\right| \\
= & -\left(E_{1}-E_{0}\right)^{2} \hat{\nu}_{r} .
\end{aligned}
$$

This relation implies that the center of mass of wave packets makes harmonic oscillations around the center $|2 n\rangle=|N\rangle$ of the oscillation region with angular velocity $\omega=E_{1}-E_{0}$, repeating the tunneling back and forth between the two Hartree states $|0\rangle$ and $|2 N\rangle$. In the above transformation of the equation we use the completeness

$$
\sum_{k=0}^{1}\left|\Psi_{k}\right\rangle\left\langle\Psi_{k}\right|=1
$$

of the eigenstates $\Psi_{k}$ in the two-eigenstate space and the relations $\left\langle\Psi_{k}\left|\hat{\nu}_{r}\right| \Psi_{k}\right\rangle=0$, which are satisfied by the eigenstates $\Psi_{k}$ that are either symmetric or antisymmetric with respect to $2 n<N$ and $2 n>N$. The amplitude of the harmonic tunneling oscillations is determined by the initial value of the coordinate of the center of mass of wave packets, $\nu_{r}(0)=\left\langle\Psi(0)\left|\hat{\nu}_{r}\right| \Psi(0)\right\rangle$, in the two-state approximation.

The evolutions of the velocity, $\dot{\nu}(t)=\langle\Psi(t)|\dot{\hat{\nu}}| \Psi(t)\rangle$, and of the acceleration, $\ddot{\nu}(t)=\langle\Psi(t)|\ddot{\nu}| \Psi(t)\rangle$, of the center of mass of the two wave packets involve the differences of the energy eigenvalues $E_{k}$, since the velocity and the accelera-
TABLE VII. The values of the acceleration $\ddot{\nu}(t)$ of the center of mass of the two wave packets in the 12-nucleon system governed by the adiabatic Hamiltonian $H_{\text {adi }}$ in Eq. (46) are calculated in the two-state approximation as a function of time $t$ in units of the period $\tau=2 \pi /\left(E_{1}-E_{0}\right)$ of the harmonic collective tunneling oscillations. Dividing the acceleration expressed on the right hand side of Eq. (65) into two terms, i.e., the potential energy term and the residual interaction term, we show the values of the two terms and the sum of the two.

\begin{tabular}{cccc}
\hline \hline$t$ & Potential & $\begin{array}{c}\text { Residual } \\
\text { interaction }\end{array}$ & Sum \\
\hline 0.00 & -7769.5 & 7779.3 & 9.8 \\
0.05 & -7389.2 & 7398.5 & 9.3 \\
0.10 & -6285.6 & 6293.6 & 7.9 \\
0.15 & -4566.8 & 4572.6 & 5.8 \\
0.20 & -2400.9 & 2403.9 & 3.0 \\
0.25 & 0.0 & 0.0 & 0.0 \\
0.30 & 2400.9 & -2403.9 & -3.0 \\
0.35 & 4566.8 & -4572.6 & -5.8 \\
0.40 & 6285.6 & -6293.6 & -7.9 \\
0.45 & 7389.2 & -7398.6 & -9.3 \\
0.50 & 7769.5 & -7779.3 & -9.8 \\
\hline \hline
\end{tabular}

tion are defined by the commutation relations of the coordinate $\hat{\nu}$ of the center of mass with the Hamiltonian $H$ as above. Some of the eigenenergy differences are so large that the velocity $\dot{\nu}(t)$ and the acceleration $\ddot{\nu}(t)$ of the center of mass, extremely affected by quantum fluctuations, oscillate quite violently. The wave function in the two-state approximation,

$$
\Psi(t)=e^{-i H t}|0\rangle=\sum_{k=0}^{1}\left|\Psi_{k}\right\rangle\left\langle\Psi_{k}\left|e^{-i E_{k} t}\right| 0\right\rangle,
$$

however, yields the velocity and acceleration to be smooth functions of time $t$ of the order of the small energy differences $E_{1}-E_{0}$ and $\left(E_{1}-E_{0}\right)^{2}$, respectively.

We have calculated the wave function $\Psi(t)$, the coordinate $\nu(t)$, the velocity $\dot{\nu}(t)$, and the acceleration $\ddot{\nu}(t)$ of the center of mass of wave packets for the system governed by the adiabatic Hamiltonian $H_{\text {adi }}$ in Eq. (46). Figure 3 demonstrates the calculated coordinate $\nu(t)$ of the center of mass of wave packets. Because of the quantum fluctuations the velocity $\dot{\nu}(t)$ and the acceleration $\ddot{\nu}(t)$ of the center of mass oscillate so violently that it is not meaningful to demonstrate their values. Only the values of the acceleration $\ddot{\nu}(t)$ smoothed by the two-state approximation are listed in Table VII. Figure 3 and Table VII show that the motion of the center of mass of wave packets calculated in the two-state approximation clearly features harmonic collective tunneling oscillations. The amplitude $a=|\nu(0)-4|=3.49$ of the harmonic tunneling oscillations is determined by the initial value of the coordinate of the center of mass of wave packets, $\nu(0)=0.51$, in the two-state approximation. 
The acceleration $\ddot{\nu}(t)$ of the center of mass of two wave packets that should be proportional to the force acting on the tunneling oscillator is divided into the two terms on the right hand side of Eq. (65), i.e., the potential energy term with $\epsilon_{n}$ and the residual interaction term with $h_{n n \pm 1} h_{n \pm 1 n}$. The first term representing the potential energies $\epsilon_{n}$ can be shown to act on the center of mass of wave packets as a repulsive force as follows. The energy differences $\epsilon_{n+1}-\epsilon_{n}$ in the potential energy term in Eq. (65) are positive for $2 n<N$ and negative for $2 n>N$. The factors $h_{n n \pm 1}$ are negative. From Table VI that lists the eigenstates $\Psi_{k}$ of $H_{\text {adi }}$, we see that the expectation values of $|2 n\rangle\langle 2(n+1)|$ and $|2(n+1)\rangle\langle 2 n|$ in the nuclear wave function $\Psi(t)$ which is split into the two wave packets are positive at any time $t$. Therefore the force due to the potential energy term in Eq. (65) acts as a repulsive force to repel the center of mass of wave packets from the center $|2 n\rangle=|N\rangle$ of the oscillation region. The force due to the second term in Eq. (65) representing the residual interactions $h_{n n \pm 1}$, however, acts as an attractive force to make the center of mass oscillate through the repulsive potential energy barrier. In fact, using the relation for $\Psi_{k}$ with $k=0$ and 1 ,

$$
\left\langle 2 \mid \Psi_{k}\right\rangle=\frac{h_{10}}{E_{k}-\epsilon_{1}}\left\langle 0 \mid \Psi_{k}\right\rangle,
$$

which is obtained in a perturbation theory, we can generally prove that the second term in Eq. (65) representing the residual interaction overcomes the first term from the potential energies $\epsilon_{n}$, concluding that the center of mass of wave packets oscillates through the potential energy barrier. Note that, if the nondiagonal matrix elements $h_{n n+1}$ and $h_{n+1 n}$ are constant independently of $n$, i.e., if the mass in the kinetic energy in the Schrödinger difference equation for a Hamiltoniain $H$, which is equivalent to the inverse of twice the matrix elements $h_{n n \pm 1}[4,9]$, is constant independently of $n$, the attractive force due to the residual interaction vanishes inside the region of the tunneling oscillation motion. Only at the edges $|0\rangle$ and $|2 N\rangle$ of the region of motion, the attractive force is brought about by the second term in Eq. (65) to steer the tunneling of the center of mass of wave packets through the potential energy barrier.

Here we briefly discuss the case of the Hamiltonian $H$ $=\kappa Q^{\dagger} Q$ with the sign opposite to the present case of the Hamiltonian $H=-\kappa Q^{\dagger} Q$. The Hamiltonian $H=\kappa Q^{\dagger} Q$ yields an attractive adiabatic potential energy well. This Hamiltonian, however, does not make much difference from the present Hamiltonian $H=-\kappa Q^{\dagger} Q$. The new Hamiltonian has the same set of eigenstates as $H=-\kappa Q^{\dagger} Q$, while the eigenenergies $E_{k}$ are of opposite signs and the order of the energy levels is reversed. The wave function $\Psi(t)$ $=e^{-i H t}|0\rangle$ for the nuclear evolution, if it starts with a deformed state $|0\rangle$, is split into two wave packets separated at the two sides of the prolate and oblate states, and no wave packets can traverse the attractive potential energy well. The current, however, flows from one wave packet to another so that the center of mass of the two wave packets can traverse the well. In a same way as the case of a potential energy barrier, the potential energy term with the energy differences $\epsilon_{n+1}-\epsilon_{n}$ multiplied by positive nondiagonal matrix elements $h_{n n \pm 1}$ on the right hand side of Eq. (65) for the acceleration $\ddot{\nu}$ acts to repel the center of mass to stay at an edge of the potential energy well, while the residual interaction term in Eq. (65) acts as an attractive force on the center of mass to oscillate over the potential energy well. Even in the case of the attractive potential energy well, if the initial state $|0\rangle$ is not coupled with the eigenstates $\Psi_{k}$ of $H$ that have probability distribution in the central region of the well, no wave packets at an edge of the region can, in general, traverse the potential energy well. Therefore, it is concluded that a quantum mechanical system expressed by a tridiagonal Hamiltonian makes collective motions contradictory to classical mechanics even in an attractive potential energy well.

\section{DISCUSSIONS AND CONCLUSION}

We have formulated the nuclear collective tunneling transitions from one Hartree minimum state to another. We reformed the field Hamiltonian used for the Hartree calculations into the Hamiltonian $H$ effective for the Hartree states and for the collective tunneling in Eq. (16), which employs the quadrupole-quadrupole interaction for the residual interaction. The Hamiltonian does not include the pairing interaction, which may play a role in the collective tunneling from the oblate state to the prolate state. The qualitative features of the present discussion might be persistent even when we take into account the pairing interaction.

We developed a real-time description for the collective tunneling. We showed that an adiabatic approximation provides a tridiagonal expression for the Hamiltonian $H$ on the basis of adiabatic $2 n$-particle- $2 n$-hole states $|2 n\rangle$. Owing to the residual interaction, the nuclear system, governed by the tridiagonal Hamiltonian symmetric between two Hartree states, coherently tunnels back and forth between the two Hartree states to make harmonic tunneling oscillations. We investigated the mechanism of the system to make the harmonic oscillations of quantum mechanical collective tunneling. While a crowd of quantum fluctuations coherently shifts back and forth in phase with the harmonic tunneling oscillations of the center of mass of wave packets, the symmetric nuclear system retains the energy for the harmonic tunneling oscillations.

The tables of isotopes show that the two lowest-lying $0^{+}$ energy levels of ${ }^{28} \mathrm{Si}$ are split by $5.0 \mathrm{MeV}$ [10]. This may suggest that the nuclear system is not precisely symmetric between the oblate state and the prolate state. If so, a more detailed description may be necessary for the investigation of the collective tunneling oscillations of the nuclear system. We will investigate the tunneling oscillation phenomena in nuclear physics in the case in which the asymmetry of the system arises. 
[1] S. Tomsovic, Phys. Scr., T 90, 162 (2001).

[2] T. Nagata, A. Kato, and T. Kohmura, Nucl. Phys. A601, 333 (1996)

[3] H. Ohta, Master's thesis, University of Tsukuba, 2000.

[4] T. Kohmura, Y. Hashimoto, H. Ohta, and M. Maruyama, Phys. Rev. C 61, 034315 (2000).

[5] T. Kohmura, Prog. Theor. Phys. 106, 471 (2001).

[6] N.R. Walet, G. DoDang, and A. Klein, Phys. Rev. C 43, 2254
(1991).

[7] J.P. Elliott, Proc. R. Soc. London, Ser. A 245, 128 (1958).

[8] D.B. Lichtenberg, Unitary Symmetry and Elementary Particles (Academic Press, New York, 1970).

[9] P. Arve, G.F. Bertsch, J.W. Negele, and G. Puddu, Phys. Rev. C 36, 2018 (1987).

[10] Tables of Isotopes, edited by C.M. Lederer and V.S. Shirley (Wiley Interscience, New York, 1978). 